\title{
Towards RGB LEDs based on rare earth-doped ZnO
}

\author{
J.L. Frieiro, ${ }^{1,2}$ C. Guillaume, ${ }^{3}$ J. López-Vidrier, ${ }^{1,2}$ O. Blázquez ${ }^{1,2}$, S. González-Torres ${ }^{1,2}$ C. \\ Labbé, ${ }^{3}$ S. Hernández, ${ }^{1,2}$ X. Portier, ${ }^{3}$ B. Garrido ${ }^{1,2}$ \\ ${ }^{1}$ MIND, Departament d'Enginyeria Electrònica i Biomèdica, Universitat de Barcelona, Martí i \\ Franquès 1, 08028 Barcelona (Spain) \\ ${ }^{2}$ Institute of Nanoscience and Nanotechnology ( $\left(\mathrm{N}^{2} \mathrm{UB}\right)$, Universitat de Barcelona, Av. Joan \\ XXIII S/N, 08028 Barcelona (Spain) \\ ${ }^{3}$ CIMAP Normandie Université, ENSICAEN, UNICAEN, CEA, UMR CNRS 6252, 6 \\ Boulevard du Maréchal Juin, 14050 Caen (France)
}

Keywords: light emitting devices, electroluminescence, rare earths, RGB, ZnO

\begin{abstract}
By using $\mathrm{ZnO}$ thin films doped with $\mathrm{Ce}, \mathrm{Tb}$ or Eu, deposited via RF magnetron sputtering, we have developed monochromatic (blue, green and red, respectively) light emitting devices. The rare earth ions introduced with doping rates lower than $2 \%$ exhibit narrow and intense emission peaks due to electronic transitions in relaxation processes induced after electrical excitation. This study proves zinc oxide to be a good host for these elements; its high conductivity and optical transparency in the visible range being as well exploited as top transparent electrode. After structural characterization of the different doped layers, a device structure with intense electroluminescence is presented, modelled, and electrically and optically characterized. The different emission spectra obtained are compared in a chromatic diagram, providing a reference for future works with similar devices. The results hereby presented demonstrate three operating monochromatic light emitting devices, as well as a combination of the three species into another one, with a simply-designed structure compatible with current Si technology and demonstrating an integrated RGB emission.
\end{abstract}

\section{Introduction}

For decades now, zinc oxide $(\mathrm{ZnO})$ has attracted much attention of a large part of the optoelectronics research community. This well-known transition metal oxide exhibits a wurzite structure, a direct bandgap with an energy of $3.4 \mathrm{eV}$ and a large exciton binding energy (60 $\mathrm{meV})$,[1] making this material suitable for many applications including thin film transistors, light emitters, detectors and gas sensors.[2,3] Its high transparency to visible light $(>80 \%)$ and good conductivity $\left(\sim 100 \Omega^{-1} \mathrm{~cm}^{-1}\right)$ have also made it a promising transparent conductive oxide (TCO), boosted in many cases by the doping of elements like $\mathrm{Al}$, which further increases its conductivity while maintaining a high transparency.[4,5] In the context of light emission, it has been compared to gallium nitride $(\mathrm{GaN})$, as they both arrange in the same crystalline structure and present very similar bandgap energy although they significantly differ in exciton binding energy, $25 \mathrm{meV}$ for $\mathrm{GaN}$, which in principle makes $\mathrm{ZnO}$ more suitable for light emission. The advantage of $\mathrm{GaN}$ over $\mathrm{ZnO}$ comes in the easier fabrication of $p$-type films, the tunability of the bandgap (and thus light emission energy) from the UV to the near IR when alloyed with In and/or $\mathrm{Al}$ (AlInGaN alloys) and the lower sensibility to defects and dislocations as non-radiative 
recombination centers.[6] Nevertheless, both the abundance of $\mathrm{Zn}$ compared to Ga and higher exciton stability of $\mathrm{ZnO}$ at room temperature, together with its non-toxicity, still make $\mathrm{ZnO}$ relevant for optoelectronic applications.

In the case of $\mathrm{ZnO}$, it is a semiconductor that intrinsically presents oxygen vacancies $\left(\mathrm{V}_{\mathrm{o}}\right)$ and zinc interstitials $\left(\mathrm{Zn}_{\mathrm{i}}\right)$ that act as donor impurities,[7] being possible to control them during the fabrication or posterior annealing processes. This particularity is of special interest for producing good $n$-type $\mathrm{ZnO}$ layers, further enhanced by doping with group-III elements such as $\mathrm{Al}, \mathrm{Ga}$ and In as substitutes for $\mathrm{Zn}$ sites or group-VII elements such as $\mathrm{Cl}$ and I as substitutes for $\mathrm{O}$ ones. Nevertheless, reliable $p$-type layers remain a major challenge because of the selfcompensation from those native donor defects. Some efforts have been done in this direction using group-I (substitutes for $\mathrm{Zn}$ ) or group-V (for O) elements, or by alloying it with Mg. [8,9] Therefore, the achievement of electroluminescence devices based on a $\mathrm{ZnO} p$ - $n$ junction still has to overcome this issue.

Due to this last limitation, different strategies for producing $\mathrm{ZnO}$-based light emitting devices (LEDs) have been researched, including defect engineering,[10] nanostructuring[11] or the doping with already optically-active centers. In this context, rare earth (RE) elements have demonstrated over decades outstanding optical and electroluminescent (EL) properties.[12] Among all these, it is especially notable the intense, stable and narrow emission they present, the different RE species covering a wide range of the electromagnetic spectrum, spanning from the near-infrared (NIR) to ultraviolet (UV).[13] Their hosting material specially affects the scattering of the emission, which is larger for polycrystalline and amorphous materials and weaker for crystalline matrices. RE elements are being employed by the industry for the fabrication of LEDs as part of phosphorescent stacks in an optical down conversion process. Additionally, the suitability of different host matrices, [12,14,15] including $\mathrm{ZnO}$, [16-19] for hosting optically-active RE dopants has been extensively proven in the past, aiming at profiting from their emission after electrical excitation.

The emission process in $\mathrm{RE}$ is due to the electronic transitions within the inner $f$-shell of these elements (from the $4^{\text {th }}$ or $5^{\text {th }}$ levels for lanthanides and actinides, respectively), leading to photon emission with an energy dependent on those transitions. Initially, RE elements have their outer $f$-shell partially filled. In a $\mathrm{C}_{4 \mathrm{v}}$ they have a +3 -valence state as a result of the loss of the two electrons from the outer $s$-shell and one from the partially filled $f$-shell. This valence state is possible in $\mathrm{ZnO}$-doped films when the $\mathrm{RE}$ ions occupy $\mathrm{Zn}$ sites.[19] In this configuration, excited lanthanide ions can relax through the predominant intra- $4 f$ (practically independent on the local environment) and $5 d$-to- $4 f$ (quite sensitive to the matrix and local environment) electronic transitions,[20-22] leading to intense narrow emission peaks.

Regarding research in the field of LEDs, of particular interest would result the finding of a redgreen-blue (RGB) system able to be employed as full-color light source. Bearing this objective in mind, in this work we have studied three of these RE elements: europium (Eu), terbium (Tb) and cerium (Ce). In Figure 1, the main electronic transitions involved in the emission of these three elements are represented.[16,23,24] Please note that the more probable transitions, thus resulting in more intense emissions, are indicated in color referring to their apparent color: blue for $\mathrm{Ce}^{3+}(464 \mathrm{~nm})$, green for $\mathrm{Tb}^{3+}(544 \mathrm{~nm})$ and red for $\mathrm{Eu}^{3+}(616 \mathrm{~nm})$. 


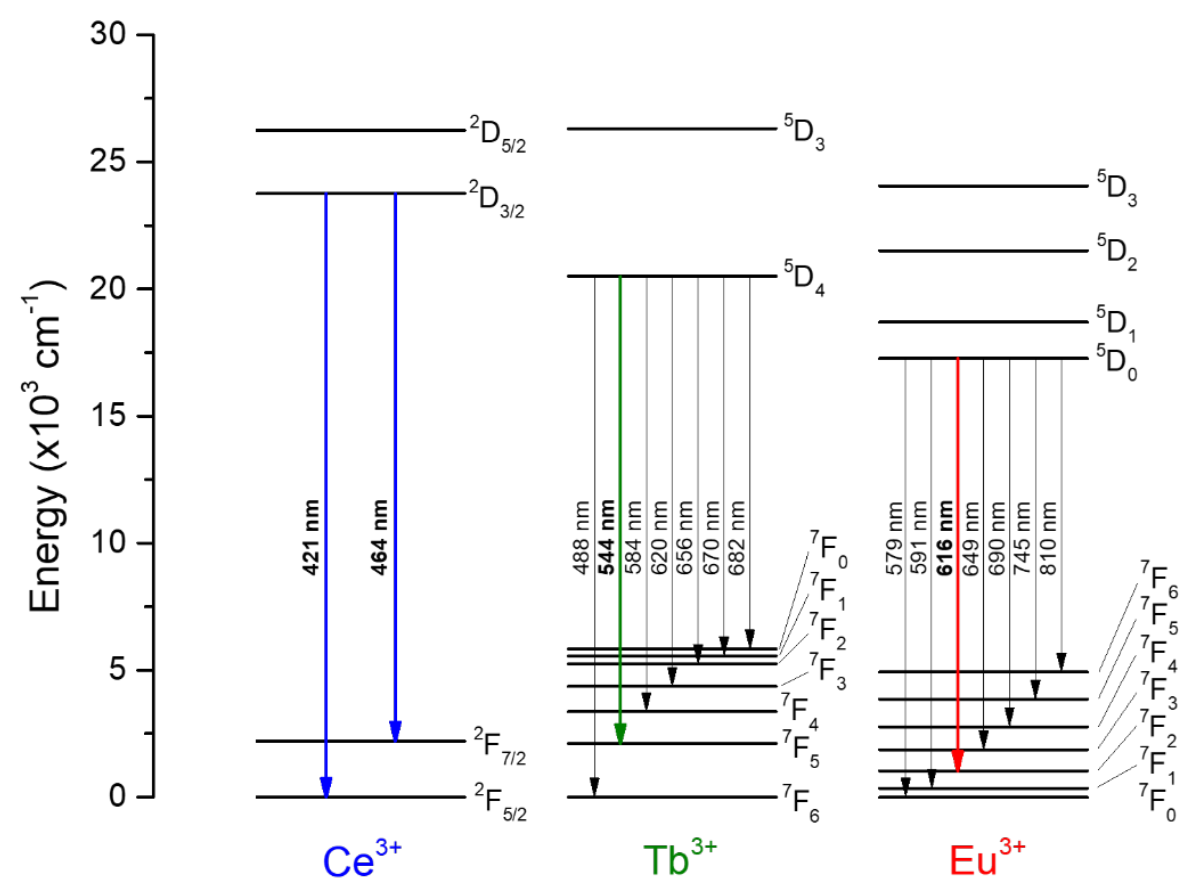

Figure 1. 5d-to-4f electronic transitions in the rare earth ions considered for this work $\left(\mathrm{Ce}^{3+}\right.$, $\mathrm{Tb}^{3+}$ and $\mathrm{Eu}^{3+}$ ) and the wavelength corresponding to the resulting photons. The main transitions have been highlighted in the schematics in blue, green and red, respectively for $\mathrm{Ce}^{3+}, \mathrm{Tb}^{3+}$ and $\mathrm{Eu}^{3+}$.

Due to the precise conditions required for the optical activation of RE ions, the doping,[17] fabrication process[25] or local environment [18,26-28] become critical factors to obtain reliable devices that take profit of their luminescent properties. In this work, we have fabricated RE-doped $\mathrm{ZnO}$ layers by radiofrequency (RF) magnetron sputtering onto $n$-type Si substrates, with an atomic-layer-deposited (ALD) $\mathrm{ZnO}$ as top transparent electrode. Three different REspecies have been employed, $\mathrm{Eu}, \mathrm{Tb}$ and $\mathrm{Ce}$, for emitting, as discussed above, in the red, green and blue colors, respectively; with doping concentrations lower than 2 at. $\%$ for each dopant. The structural properties have been characterized by transmission electron microscopy (TEM) and energy dispersive X-ray (EDX) spectroscopy, allowing determining the spatial distribution of the RE-ions at the nanoscale. Electrical characterization has revealed different conduction mechanisms that can be related to the structural properties of the layers and the device configuration. Finally, all the studied devices exhibit EL as a consequence of the excitation of the RE-species. The obtained emissions are discussed in terms of the different RE species, the structural and electrical properties. The results here presented get us closer to achieving a tunable RGB-color LED fabricated with a base on abundant materials such as Si and Zn, taking advantage of the emission from RE ions as optical centers.

\section{Materials and methods}

The $\mathrm{ZnO}$ films were deposited from doped $\mathrm{ZnO}$ targets on a $n$-type (100)-oriented Si substrate via a 3-inch RF magnetron sputtering system, using a power density of $0.96 \mathrm{~W} \cdot \mathrm{cm}^{-2}, 15 \mu$ bar of pressure, Ar flux of $2 \mathrm{sccm}$, and at a substrate temperature of $400{ }^{\circ} \mathrm{C}$. To avoid crosscontamination, three different $\mathrm{ZnO}$ targets nominally identical were used for each of the three different dopant species. Only two targets could be loaded in the chamber. Doping was obtained by placing $\mathrm{RE}$ oxide pellets $\left(\mathrm{CeO}_{2}, \mathrm{~Tb}_{4} \mathrm{O}_{7}\right.$ or $\left.\mathrm{Eu}_{2} \mathrm{O}_{3}\right)$ on the top of each $\mathrm{ZnO}$ target for the 
deposition of the films. Under these conditions, three 60-nm-thick single-layer samples were deposited: (i) $\mathrm{ZnO}: \mathrm{Eu}, \mathrm{Tb}$, (ii) $\mathrm{ZnO}: \mathrm{Tb}$, and (iii) $\mathrm{ZnO}: \mathrm{Ce}$. An additional sample containing the three RE species was fabricated by consecutively depositing 20 -nm-thick layers of the three mentioned films in a (iv) $\mathrm{ZnO}: \mathrm{Ce} / \mathrm{ZnO}: \mathrm{Tb} / \mathrm{ZnO}: \mathrm{Eu}, \mathrm{Tb}$ structure. For this three-layer structure, the sputtering chamber contained initially two targets which could not be activated simultaneously. First, a $\mathrm{ZnO}: \mathrm{Tb}$,Eu layer was deposited and then the $\mathrm{ZnO}: \mathrm{Tb}$ layer using the second target. For the last $\mathrm{ZnO}$ :Ce layer, the chamber was opened to replace one of the two targets. The RE doping concentration was $\sim 1.7$ at.\% in $\mathrm{ZnO}: \mathrm{Ce}, \sim 2$ at. $\%$ in $\mathrm{ZnO}: \mathrm{Tb}$, and 0.8 at. $\%$ of $\mathrm{Eu}$ and $\sim 1.6$ at.\% of $\mathrm{Tb}$ in $\mathrm{ZnO}: \mathrm{Eu}, \mathrm{Tb}$, as determined by EDX measurements on $2 \mu \mathrm{m}$ thick layers in a scanning electron microscope. Afterwards, all samples underwent an annealing process for 1 hour at $700{ }^{\circ} \mathrm{C}$ in a $\mathrm{N}_{2}$ atmosphere.

Structural characterization by electron microscopy was done in sample (iv), as it can provide representative information of the deposition for the three films under investigation. The foils were prepared by focused ion beam (FEI HeliosNanolab 660), and their structure was analyzed at the nanoscale by conventional and high resolution TEM (HRTEM) using a double-corrected cold FEG ARM200F JEOL microscope, operated at $200 \mathrm{kV}$ and equipped with a high-angle annular dark field (HAADF) detector and an EDX Centurio spectrometer. EDX profiles were acquired along a 75-nm straight line across the film with 120 probed spots and 1 second acquisition time per spot.

The top 100-nm-thick $\mathrm{ZnO}$ electrode was deposited by means of atomic layer deposition (ALD) at a deposition temperature of $200{ }^{\circ} \mathrm{C}$, resulting in a highly-conductive layer (conductivity $>100$ $\Omega^{-1} \mathrm{~cm}^{-1}$ ) with an optical transparency of $\sim 85 \%$ through the visible range.[4] Afterwards, circular contacts with $500 \mu \mathrm{m}$ diameter were patterned via conventional photolithography. Finally, a full-area deposition by electron beam evaporation (EBE) of $\mathrm{Al}$ created the common bottom electrode to finalize the devices.

Current-voltage $[I(V)]$ measurements were carried out placing the devices within a Cascade Microtech Summit 11000 probe station coupled to an Agilent B1500A semiconductor device analyzer. A Faraday cage is used to isolate the devices from external electromagnetic noise. In all electrical measurements, a voltage was applied on the top $\mathrm{ZnO}$ contact while the bottom $\mathrm{Al}$ was grounded through the chuck of the system. The voltage applied this way was swept in steps of $50 \mathrm{mV} \cdot \mathrm{s}^{-1}$. EL emission from the devices was collected by a microscope with a $20 \times \mathrm{NIR}$ objective with a numerical aperture of 0.4 , and it was spectrally resolved via a $1 / 4 \mathrm{~m}$ monochromator and a LN-cooled charge coupled device (CCD) in the $400 \mathrm{~nm}$ to $1000 \mathrm{~nm}$ range. Integrated EL was also collected by replacing the monochromator-CCD system by a GaAs photomultiplier tube (PMT), sensitive in the range from $350 \mathrm{~nm}$ to $900 \mathrm{~nm}$.

\section{Results}

\section{a. Structural characterization}

Figure 2 shows a HRTEM image (Figure 2a) of sample (iv) and the EDX analysis performed (Figure 2b) for the elements present in the sample along the growth direction, as indicated by the line in the HRTEM image. In Figure 2a, different regions on the sample can be clearly identified. From the bottom, it can first be found the $n$-type Si substrate with a $\sim 5$-nm layer of native $\mathrm{SiO}_{\mathrm{x}}$ on top, which was intentionally left unremoved before the deposition process to be used as electron energy barrier in electrical measurements. The deposited RE-doped $\mathrm{ZnO}$ films can be observed above the $\mathrm{SiO}_{\mathrm{x}}$. Please note here that there is no indication of the existence of any interface between each $\mathrm{ZnO}$ layer, despite the different steps for depositing all $\mathrm{ZnO}$ layers. However, non-uniform contrast zones are clearly visible suggesting local variations of the 
Bragg conditions due to local strains induced by the presence of dopants in the $\mathrm{ZnO}$ matrix. On top of the stack, a carbon layer of a few nanometers and Pt were deposited in order to protect the top surface of the stack, before TEM sample preparation by FIB. The doped-ZnO total thickness was measured to be $\sim 65 \mathrm{~nm}$ and its polycrystalline nature can be clearly distinguished in the image by the different crystalline plane orientations present in the film. Some artifacts due to Moiré patterns can also be observed in the image.

To assess the composition of the layers upon annealing, EDX measurements were done along the vertical line indicated in Figure 2a. The atomic profiles corresponding to Pt (black), Si (grey), $\mathrm{Ce}$ (blue), Tb (green) and Eu (red) are plotted in Figure 2b. As shown in the TEM image, $\mathrm{Si}$ is only present at the bottom of the sample, whereas Pt is only present on top. To compare the concentration of each RE element for each sublayer, vertical lines have been drawn approximately where the profiles of the elements change, therefore suggesting the interface between the different sublayers. The intended nominal thickness for each sublayer was $20 \mathrm{~nm}$. However, the actual thickness slightly differ; in particular, it can be observed in the TEM image that the Ce-doped layer is $\sim 8 \mathrm{~nm}$ thinner than the Tb-doped and $\mathrm{Tb}$ - and Eu-co-doped ones.

The EDX results also reveal the non-homogeneity of the RE ions within the layers but they allow positioning approximately the various interfaces between the sublayers. Despite uncertainty of the measurements (about 1 at.\%), fluctuation of the signals where each RE doping was nominally intended is indicative of their presence within the $\mathrm{ZnO}$. This is particularly the case of $\mathrm{Tb}$ whose concentration reaches about 2 at.\% in the middle of the structure. Thus, while $\mathrm{Tb}$ is uniformly distributed along the $\mathrm{ZnO}: \mathrm{Tb}$ layer in the middle of the structure, the other two layers present a high agglomeration of the RE ions in certain zones: on one hand, $\mathrm{Ce}$ is accumulated at the top of the third layer, in a small region of $\sim 5 \mathrm{~nm}$ with a narrow depleted zone just below; on the other hand, in the first layer, Tb profile presents also a depleted region close to the interface with $\mathrm{SiO}_{\mathrm{x}}$ where both $\mathrm{Tb}$ and Eu have accumulated very significantly in a region of $\sim 8 \mathrm{~nm}$. Indeed, this latter fact was corroborated with a HRTEM image (see Figure 2c, corresponding to the squared region in Figure 2a) of the bottom of the substrate, where a thin $(\sim 2-3 \mathrm{~nm})$ amorphous layer enriched in $\mathrm{Tb}$ and Eu was identified on top of the $\mathrm{SiO}_{\mathrm{x}}$ layer.

(a)

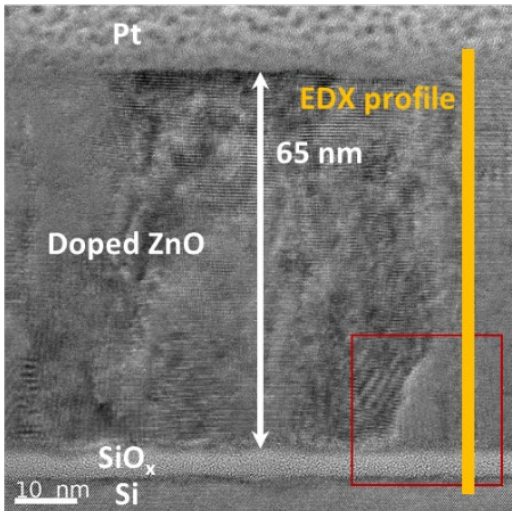



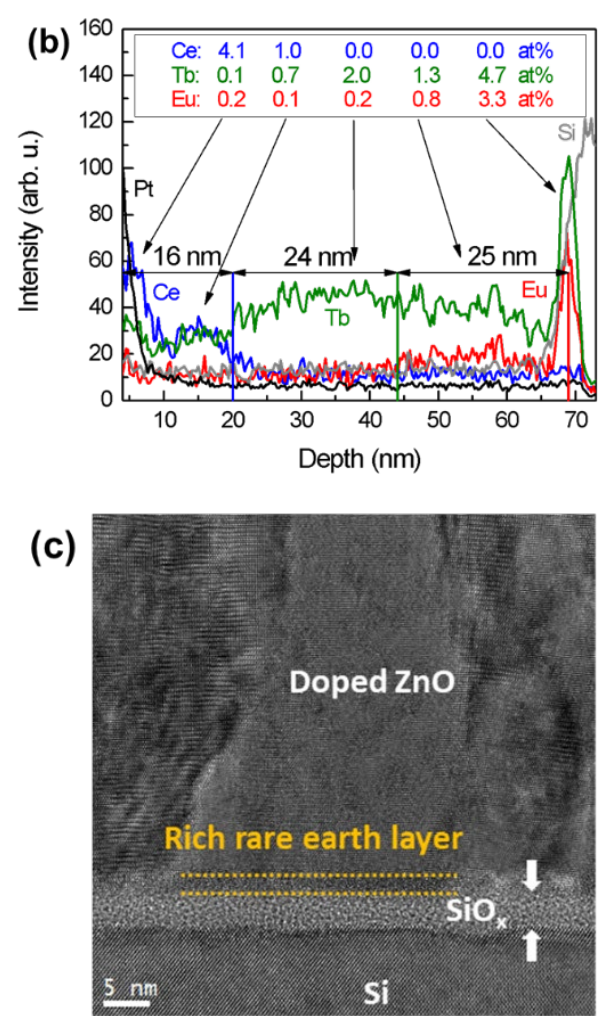

Figure 2. (a) TEM image corresponding to the deposited sample containing the $\mathrm{ZnO}: \mathrm{Ce} / \mathrm{ZnO}: \mathrm{Tb} / \mathrm{ZnO}: \mathrm{Eu}, \mathrm{Tb}$ stack, the total stack thickness measured being $\sim 65 \mathrm{~nm}$. (b) EDX depth profiles for $\mathrm{Pt}, \mathrm{Si}, \mathrm{Ce}, \mathrm{Tb}$ and $\mathrm{Eu}$, obtained through 120 points measured along the $75 \mathrm{~nm}$ vertical line indicated in (a). (c) HRTEM image of the bottom of the structure, corresponding to the red square in (a), where $\mathrm{Tb}$ and Eu have accumulated near the $\mathrm{SiO}_{\mathrm{x}}$ layer.

\section{b. Electrical characterization}

Initially, an electrical characterization via $I(V)$ measurements was performed in order to understand the conduction regime governing each single-layer device. Figure 3 displays the $I(V)$ curves for the $\mathrm{ZnO}: \mathrm{Ce}$ (blue), $\mathrm{ZnO}: \mathrm{Tb}$ (green), $\mathrm{ZnO}: \mathrm{Eu}, \mathrm{Tb}$ (red) and $\mathrm{ZnO}: \mathrm{Ce} / \mathrm{ZnO}: \mathrm{Tb} / \mathrm{ZnO}: \mathrm{Eu}, \mathrm{Tb}$ (orange) devices in a range from $-8 \mathrm{~V}$ to $10 \mathrm{~V}$. A sketch of the devices structure and their electrical connections during measurements is displayed in the inset at the bottom right corner of Figure 3a. At a first glance, Figure 3a reveals a large rectification behavior in all four samples, exhibiting well-differentiated current level under forward and reverse biasing ( $V>0$ and $V<0$, respectively). This rectification is a consequence of the heterojunction formed by the different layers composing the device structure, and it is directly correlated with its energy band diagram. For forward bias, both $\mathrm{ZnO}: \mathrm{Tb}$ and $\mathrm{ZnO}: \mathrm{Eu}, \mathrm{Tb}$ devices show a similar conduction behavior, resembled at one order of magnitude lower by $\mathrm{ZnO}: \mathrm{Ce} / \mathrm{ZnO}: \mathrm{Tb} / \mathrm{ZnO}: \mathrm{Eu}, \mathrm{Tb}$ and slightly different from the line-shape exhibited by $\mathrm{ZnO}: \mathrm{Ce}$. In addition, all four device structures present a pronounced change in the $I(V)$ curve around $7 \mathrm{~V}$, that could be associated with a change in the carrier transport through the structure as will be further covered. 

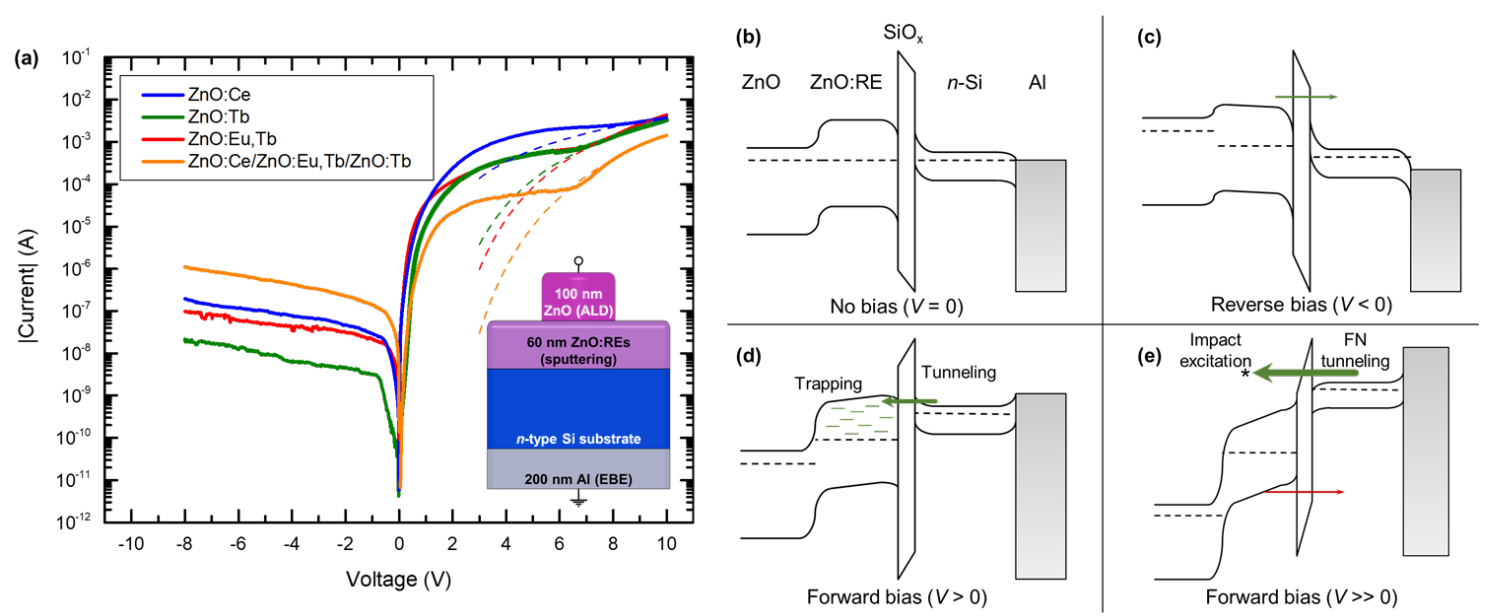

Figure 3. (a) $I(V)$ curves corresponding to the devices with $\mathrm{ZnO}: \mathrm{Ce}, \mathrm{ZnO}: \mathrm{Tb}$ and $\mathrm{ZnO}: \mathrm{Eu}, \mathrm{Tb}$ active layers in the range from $-8 \mathrm{~V}$ to $10 \mathrm{~V}$, respectively represented with a blue, green, red and orange line. Dashed lines present a Fowler-Nordheim fitting for each curve at high voltages. Inset sketch shows the structure of the devices under study. (b) Schematic energy band diagram for the device structure, with each layer labelled, under no applied bias. (c) Modified energy band diagrams under the application of reverse bias. (d), (e) Band diagrams under low and high forward biasing, respectively. Green and red arrows indicate the direction of the injected electrons and holes, respectively.

The observed trends can be explained in terms of the energy band diagrams of our devices. Bearing in mind our device configuration and the electronic band structure of the different layers, we have depicted in Figures $3 b, c, d$ and e, the energy band diagrams for the device structure under four different bias conditions: (b) no bias, (c) reverse and (d) forward bias $(V>$ 0 ), (e) high forward bias $(V>>0)$. The top $\mathrm{ZnO}$ electrode and the $n$-type substrate present high electronic densities, with the energy Fermi level close to the bottom of the conduction band, whereas the RE-doped $\mathrm{ZnO}$ has been considered an intrinsic (or quasi-intrinsic) semiconductor, with the energy Fermi level practically in the middle of the band gap, as the introduction of RE dopants maintain the dielectric nature of the host matrices.[29,30] Under no bias conditions (see Figure $3 b$ ), the energy Fermi levels are aligned along the whole structure, having carriers blocked by two energy barriers at both sides of the $\mathrm{SiO}_{\mathrm{x}}$ dielectric layer: a depletion region at the $\mathrm{SiO}_{\mathrm{x}}-\mathrm{Si}$ interface and an accumulation of electrons at the $\mathrm{ZnO}^{-\mathrm{SiO}_{\mathrm{x}}}$ interface. When the devices are set under reverse bias $(V<0)$, the current rapidly increases up to $1 \mathrm{~V}$. From this point the extracted current starts to be limited (see Figure 3a). Below $1 \mathrm{~V}$, the applied voltage is weak for promoting efficient tunneling but it contributes to the extraction of carriers from traps and defects, leading to a high current increase. Further increasing the applied voltage, as it can be seen in Figure 3c, maintains the dielectric barrier of $\mathrm{SiO}_{\mathrm{x}}$ while increasing the depletion region in the Si substrate. This is partially compensated by an increase of the accumulation region in the $\mathrm{ZnO}$ layer. The overall result is that the barrier scales with the applied external voltage, allowing some electrons to tunnel through the barrier. As the applied bias increases in absolute value, the number of electrons with enough energy to tunnel also increases, thus resulting in the slight enhancement in the measured current in agreement with our experimental observations.

On the other hand, the devices present a rapid growth in current under forward bias $(V>0)$. In

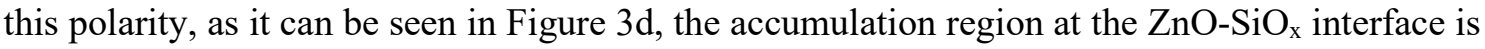
reduced until it soon shifts to a depletion region. As well, the depletion region initially present at the $\mathrm{SiO}_{\mathrm{x}}-\mathrm{Si}$ interface is rapidly reduced with the applied voltage. The combination of these 
two effects taking place allows for a faster rate of electrons to tunnel through the $\mathrm{SiO}_{\mathrm{x}}$ barrier from the Si substrate to the RE-doped $\mathrm{ZnO}$. It should be noted here that the doped-ZnO layer is polycrystalline and considered quasi-intrinsic, thus it may contain a large number of inter-band gap traps due to defects. At very low voltages $(V<1.6 \mathrm{~V})$ charge transport is again associated to carrier extraction from traps and defects (as previously identified for reverse bias conditions). As voltage increases and tunneling through the dielectric is enabled $(1.6 \mathrm{~V}<V<7 \mathrm{~V})$, a similar behavior to ohmic transport is observed due to a hopping mechanism of the electrons through the traps in the doped $\mathrm{ZnO}$ band gap, being the current bulk-limited. With the increase in applied external voltage the traps are rapidly filled and current increases until reaching a trapfilled limit, after which the current becomes limited due to space charge.

From the $I(V)$ curves, it is evident that there is a change in conduction above $7 \mathrm{~V}$ for all devices. High applied external voltages can induce an accumulation region at the $\mathrm{SiO}_{\mathrm{x}}-\mathrm{Si}$ interface, once the depletion region is completely compensated (see Figure 3e), whilst the depletion region at the $\mathrm{ZnO}-\mathrm{SiO}_{x}$ interface increases. Under these conditions, the energy bands of $\mathrm{SiO}_{\mathrm{x}}$ dielectric layer is also bent and electrons are thus able to tunnel through a reduced triangular barrier following a Fowler-Nordheim (FN) mechanism. Assuming that FN is the main conduction mechanism in this regime, the $I(V)$ curves at high voltages should follow:

$$
I=a V^{2} e^{(b / V),}
$$

where $a$ and $b$ are constants. Indeed, we have fitted all $I(V)$ curves by this mechanism (see dashed lines in Figure 3a), showing and excellent agreement with the experimental data.

In order to estimate the minimum voltage for FN conduction, we have taken into account the energy band diagram of the device structure in this range of voltages (see Figure $3 \mathrm{e}$ ) and evaluated its series resistance. To this end, some considerations have been made, treating the $\mathrm{SiO}_{\mathrm{x}}$ barrier as $\mathrm{SiO}_{2}$ : (i) Flat band voltage can be calculated supposing that the Fermi level of $n$ $\mathrm{Si}$ is close to the conduction band and the $\mathrm{ZnO}$-Fermi level in the middle of its band gap (at 1.70 $\mathrm{eV}$ from the bottom of the conduction band). Adding the electron affinity difference between $\mathrm{ZnO}$ and $\mathrm{Si}$ (4.50 and $4.05 \mathrm{eV}$, respectively), it results in a flat band voltage of $2.15 \mathrm{~V}$. (ii) The minimum voltage required for overcoming the offset energy between $\mathrm{Si}$ and $\mathrm{SiO}_{2}$ tunneling from the $\mathrm{Si}$ conduction band is equal to the difference in their electron affinities $(4.05 \mathrm{eV}$ for $\mathrm{Si}$ and $0.90 \mathrm{eV}$ for $\mathrm{SiO}_{2}$ ), that is, $3.15 \mathrm{eV}$. (iii) Finally, the series resistance (and thus the voltage drop) can be numerically evaluated for each $I(V)$ curve within the FN regime, using an iterative method. The resulting values from this procedure are summarized in Table I. Adding these three terms we can estimate the minimum voltages for FN conduction in each device as it appears in the last column of Table I. The FN fittings shown in Figure 3a employ this value as lower limit for the fitting range.

Table I. Calculation of the minimum external voltage required to enter the FN tunneling regime.

\begin{tabular}{|c|c|c|c|c|c|}
\hline Device & $\begin{array}{c}\text { Flat- } \\
\text { band } \\
\text { voltage } \\
(\mathrm{V})\end{array}$ & $\begin{array}{c}\mathrm{SiO}_{2} \\
\text { bending } \\
\text { voltage } \\
(\mathrm{V}) \\
\end{array}$ & $\begin{array}{c}\text { Series } \\
\text { resistance } \\
\left(\mathrm{R}_{\mathrm{s}}, \text { in } \mathrm{FN}\right) \\
(\mathrm{k} \Omega) \\
\end{array}$ & $\begin{array}{c}\text { Voltage } \\
\text { drop } \\
\text { (due to } \\
\left.\mathrm{R}_{\mathrm{s}}\right)(\mathrm{V}) \\
\end{array}$ & $\begin{array}{c}\text { Minimum } \\
\text { voltage for } \\
\text { FN (V) }\end{array}$ \\
\hline $\mathrm{ZnO}: \mathrm{Ce}$ & \multirow{4}{*}{2.15} & \multirow{4}{*}{3.15} & 1.21 & 3.32 & 8.62 \\
\hline $\mathrm{ZnO}: \mathrm{Tb}$ & & & 1.79 & 1.13 & 6.43 \\
\hline ZnO:Eu,Tb & & & 1.51 & 0.7 & 6.00 \\
\hline ZnO:Ce/ZnO:Tb/ZnO:Eu,Tb & & & 10.9 & 0.79 & 6.09 \\
\hline
\end{tabular}


As previously commented, the conduction under forward bias slightly differs for $\mathrm{ZnO}$ :Ce when compared to the other two films. This is probably due to the larger concentration of $\mathrm{Ce}$ with respect to the other two RE elements in their respective films, added to an easier oxidation process.[31,32] These two facts could result in a combination of large clustering of $\mathrm{Ce}^{3+}$ and oxidation to $\mathrm{Ce}^{4+}$, generating additional defect-related traps within the $\mathrm{ZnO}$ layer that require a larger external applied voltage to be filled. In addition, the clustering of $\mathrm{Ce}$ is in line with the EDX measurements, which showed an 8-nm thinner Ce film in the tri-layer system compared to the other two. Nevertheless, once the traps are filled FN dominates the conduction, in this case, for $V>8.62 \mathrm{~V}$. For devices with the $\mathrm{ZnO}: \mathrm{Ce} / \mathrm{ZnO}: \mathrm{Tb} / \mathrm{ZnO}: \mathrm{Eu}, \mathrm{Tb}$ stack, the deposition of multiple layers generates additional grain boundaries and/or strains due to the various doping rates from one layer to the adjacent ones, which can be directly related to the increase in the series resistance. While conduction is still dominated by FN at high voltages due to the presence of traps in the doped-ZnO layer, their higher series resistance hinders the conductivity by one order of magnitude.

\section{c. Electroluminescence emission}

In all devices, emission has only been observed under forward bias, in agreement with the higher charge injection under this condition, as determined by $I(V)$ measurements. The complete analysis on the EL properties of the RE-doped $\mathrm{ZnO}$ devices is detailed in Figure 4. Prior to spectrally resolving the EL of the devices, its integrated collection helped identifying the emission threshold and the relation of the emission with current, and thus the applied external voltage. For these measurements, the applied voltage was swept from 0 to $8 \mathrm{~V}$ in steps of 0.1 $\mathrm{V} \cdot \mathrm{s}^{-1}$. The results of this study are displayed in Figure $4 \mathrm{a}$, following the color scheme of the devices analyzed in Figure 3a. Both $\mathrm{ZnO}: \mathrm{Tb}$ and $\mathrm{ZnO}: \mathrm{Eu}, \mathrm{Tb}$ present again similar behaviors, with emission thresholds at 200-500 $\mu \mathrm{A}$. This threshold is increased to $1 \mathrm{~mA}$ in $\mathrm{ZnO}$ :Ce, thus indicating a less efficient emission. In the case of the multilayer device, the threshold is reduced to $10 \mu \mathrm{A}$, pointing to a higher efficiency when close to the threshold that could be related to the increased series resistance maintaining $\mathrm{FN}$ as main electron injection mechanism. It must also be noted that the obtained threshold currents of single-layer devices are in accordance with the change in conduction observed at around $7 \mathrm{~V}$ (see Table I).

So far, our results present a clear correlation between the emission and the conduction regime of the device. Considering electrons as majority carriers through the system (as suggested by the energy band diagrams in Figures $3 c$ and $d$ ), the excitation mechanism of the RE ions must be related to their injection into the doped-ZnO layer. Electrons, under forward bias, are injected from the $n$-type $\mathrm{Si}$ substrate into the doped-ZnO layer by tunneling through the dielectric $\mathrm{SiO}_{\mathrm{x}}$ barrier with an energy excess equal to the difference in energy between the substrate and $\mathrm{ZnO}: \mathrm{RE}$ conduction bands. Below the threshold of FN injection, electrons gain enough energy to tunnel but are then trapped by $\mathrm{ZnO}$ inter-band gap traps, thus not being able to excite the rare earth ions. Under large applied voltage, these hot electrons might gain additional energy due to the inclination of the $\mathrm{ZnO}$ conduction band and impact with the RE ions dispersed within the $\mathrm{ZnO}: \mathrm{RE}$ layer, transferring their energy to excite the outer shell electrons of these elements, in a process called impact excitation.[30] Another (less probable) possibility includes a low injection of holes as minority carriers allowing the combination with electrons, thus creating excitons. The energy of this particle could be transferred to the RE ions thanks to the lattice.[33] Their later relaxation results in the emission of photons we detect as EL emission. In contrast and as previously discussed, under reverse bias the injection of both electrons and holes is highly quenched, diminishing the possibility of RE excitation and resulting in no-observable EL emission. 
EL emission from the devices is visible by the naked eye at high voltages. The insets in Figure 4a show pictures of this visible emission from the devices while being electrically excited (each picture is marked with a symbol that matches the graph legend). These were acquired by setting a standard photographic-digital camera focusing through the microscope and collecting the image for $30 \mathrm{~s}$ while applying $2 \mathrm{~mA}$ of constant current to the devices. Both $\mathrm{ZnO}: \mathrm{Ce}$ and the multilayered $\mathrm{ZnO}: \mathrm{Ce} / \mathrm{ZnO}: \mathrm{Tb} / \mathrm{ZnO}: \mathrm{Eu}, \mathrm{Tb}$ devices show a weak emission mainly located close to the contact point of the tip, whereas the other two exhibit a more homogeneous and intense emission, in agreement with the previous observations of the integrated EL curves. Finally, regarding the EL color exhibited by each device, Ce-doped ones present a white-blueish emission, Tb-doped devices a clear green one, Eu,Tb-doped devices emit in orange-red. Finally, the multilayered devices present a white emission at the tip location.
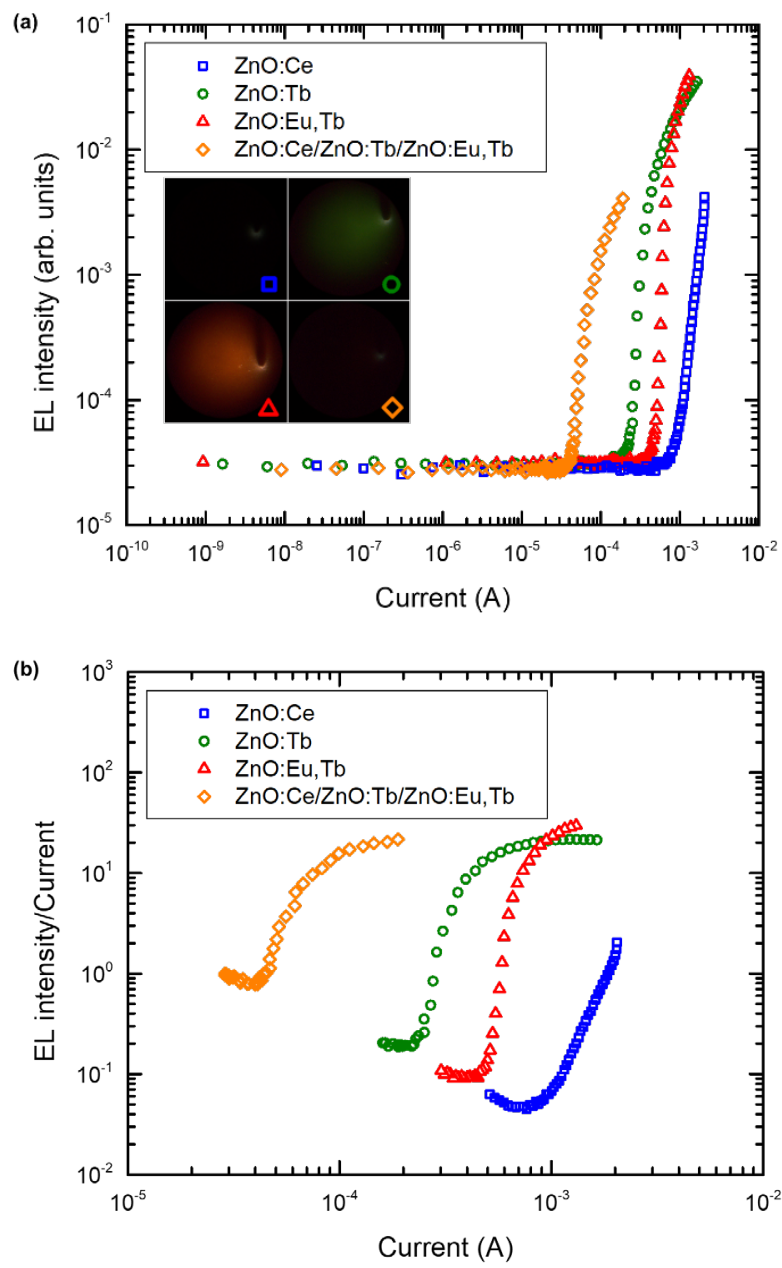


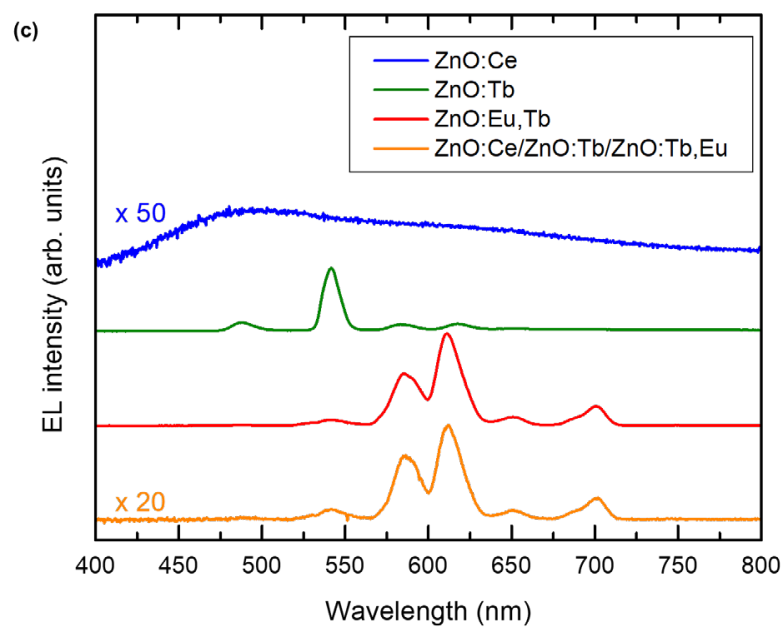

Figure 4. (a) Integrated electroluminescence of the four analyzed devices measured from 0 to 8 V. Pictures of the devices visible emission under electrical excitation are shown in the inset. (b) Representation of the integrated emission as emission efficiency, equivalent to an external quantum efficiency (EQE). (c) Corresponding electroluminescence spectra for the devices, collected during 30 seconds at $1 \mathrm{~mA}$ of applied current. Both graphs employ the same color code: $\mathrm{ZnO}: \mathrm{Ce}$ in blue, $\mathrm{ZnO}: \mathrm{Tb}$ in green, $\mathrm{ZnO}: \mathrm{Eu}, \mathrm{Tb}$ in red and $\mathrm{ZnO}: \mathrm{Ce} / \mathrm{ZnO}: \mathrm{Tb} / \mathrm{ZnO}: \mathrm{Eu}, \mathrm{Tb}$ in orange.

The representation of the EL intensity data as shown in Figure $4 \mathrm{~b}$ allows comparing the devices in terms of their emission efficiency. According to this figure of merit, the multilayered device allows attaining an optical output at much lower currents. Nevertheless, all devices present a fast increase in efficiency that is afterwards saturated, in accordance with electron injection following the $\mathrm{FN}$ regime previously identified. In the case of $\mathrm{ZnO}$ : $\mathrm{Ce}$, this regime has not yet been reached, thus resulting in the very low efficiency measured.

Once the threshold current for EL to occur was determined, the excitation mechanism has been identified and there are evidences that the emission could be seen by the naked eye, spectral measurements were carried out on each device by applying a fixed excitation current of $1 \mathrm{~mA}$ in substrate accumulation conditions. This current value corresponds a high externally applied voltage where the high-voltage FN conduction mechanism is well stablished in all devices. In Figure 4c, the EL spectra for all four devices, measured under said conditions, are displayed.

Out of the three single-doped samples, $\mathrm{ZnO}$ :Ce shows a spectrum composed of two weak and wide features centered below $500 \mathrm{~nm}$ and around $\sim 650 \mathrm{~nm}$, which would correspond to a combination of the electronic transitions listed in Figure 1 for $\mathrm{Ce}^{3+}(421$ and $464 \mathrm{~nm})$ and the emission of $\mathrm{ZnO}$ defects, respectively. The broad emission band obtained instead of the welldefined electronic transitions could be related to the electronic states delocalization because of orbital distortion, caused by $\mathrm{Ce}^{3+}$ ions clustering, as well as additional interactions with $\mathrm{Ce}^{4+}$ ions, which we have previously attributed to affect the electrical properties.[31,32] The combination of these two facts increases the probability of non-radiative processes, thus reducing the emission of the device. Oxidation of $\mathrm{Ce}^{3+}$ to $\mathrm{Ce}^{4+}$ is also compatible with the appearance of defects-related emission of $\mathrm{ZnO}$, resulting from a larger concentration of oxygen vacancies in the film.

In contrast, both $\mathrm{ZnO}: \mathrm{Tb}$ and $\mathrm{ZnO}: \mathrm{Eu}, \mathrm{Tb}$ devices show intense emission with narrow peaks corresponding to the electronic transitions of $\mathrm{Tb}^{3+}$ and $\mathrm{Eu}^{3+}$ (see Figure 1). The main emission of the $\mathrm{Tb}$-doped sample is peaked at $541 \mathrm{~nm}$, with three secondary peaks centered at $488 \mathrm{~nm}$, 
$584 \mathrm{~nm}$ and $617 \mathrm{~nm}$, all four features constituting the expected emission of $\mathrm{Tb}^{3+}$ ions.[34-36] In the case of the $\mathrm{ZnO}: \mathrm{Eu}, \mathrm{Tb}$ device, other works in the literature have proved a higher efficiency of EL emission of $\mathrm{Eu}^{3+}$ ions for the co-doped films when compared to a device only containing Eu.[34,37] Its EL exhibits its main emission peak at $611 \mathrm{~nm}$, and three secondary peaks at 585 $\mathrm{nm}, 651 \mathrm{~nm}$ and $700 \mathrm{~nm}$, which is in agreement with the Eu ${ }^{3+}$ ions emission, [38] although $\mathrm{Tb}$ is also optically active. Notwithstanding, the emission of $\mathrm{Tb}^{3+}$ ions appears to be quenched when compared to the emission of $\mathrm{Eu}^{3+}$ ones. On one hand, the main emission of $\mathrm{Tb}^{3+},{ }^{5} \mathrm{D}_{4} \rightarrow{ }^{7} \mathrm{~F}_{5}$, has a higher energy than the ${ }^{7} \mathrm{~F}_{0} \rightarrow{ }^{5} \mathrm{D}_{0}$ excitation transition of $\mathrm{Eu}^{3+}$ and thus the energy involved in this emission may be partially transferred to $\mathrm{Eu}^{3+}$ ions and converted into an $\mathrm{Eu}^{3+}$ emission (the ${ }^{5} \mathrm{D}_{1}$ and ${ }^{5} \mathrm{D}_{0}$ electronic states might be populated). On the other hand, the remaining $\mathrm{Tb}^{3+}$-related emission is masked by the emission of $\mathrm{Eu}^{3+}$, as some of the main contributions almost overlap in wavelength (see Figure 1). All these three emissions correspond well (in color and intensity) to the images of the devices shown in the insets of Figure 4a.

In agreement with the emission of the single-doped samples, the fourth one, combining all three layers, shows the characteristic emission peaks of $\mathrm{Tb}^{3+}$ and $\mathrm{Eu}^{3+}$ in the same positions as previously observed. This fact indicates that the optical activity of RE ions is still preserved when sputtered on a stack structure. $\mathrm{Tb}^{3+}$ ions emission can be detected by visually comparing the relative intensities between peaks at $541 \mathrm{~nm}$ and $611 \mathrm{~nm}$ with those in sample $\mathrm{ZnO}: \mathrm{Eu}, \mathrm{Tb}$. Therefore, the layer sequence is optimal for obtaining a combined emission of the RE ions from one device, avoiding the $\mathrm{Eu}^{3+}$ absorption of $\mathrm{Tb}^{3+}$-emitted photons that would take place if $\mathrm{Eu}$ was on top. Last, it is worth noting that the emission of the combined-RE stack is 20 times less intense (as indicated) than that of the previously commented devices, which is a consequence of the reduction in thickness of the two emitting layers (as discussed during the structural characterization), and the presence of $\mathrm{ZnO}$ :Ce on top quenching the emission from the bottom layers. The analyzed EL peaks have a FWHM of about $15 \mathrm{~nm}$, in agreement with other reported works concerning RE-doped $\mathrm{ZnO}$ electroluminescent devices.[14,15,22,34]

\section{d. Chromatic diagram}

To complement the electro-optical study carried out in this work, the resulting EL emission from the investigated devices was analyzed under the CIE 1931 standard for reference. The position of the four obtained spectra are displayed in the chromatic diagram in Figure 5: $\mathrm{ZnO}: \mathrm{Ce}$ corresponds to yellow due mainly to the emission of $\mathrm{ZnO}$ defects. Emission from $\mathrm{ZnO}: \mathrm{Tb}$ and $\mathrm{ZnO}: \mathrm{Eu}, \mathrm{Tb}$ well correspond to green and red-orange colors in the diagram, respectively. In addition, the device containing the three different layers exhibits an orange color closer to the white center of the diagram, in agreement with the presence of both $\mathrm{Tb}^{3+}$ and $\mathrm{Eu}^{3+}$ emissions. 


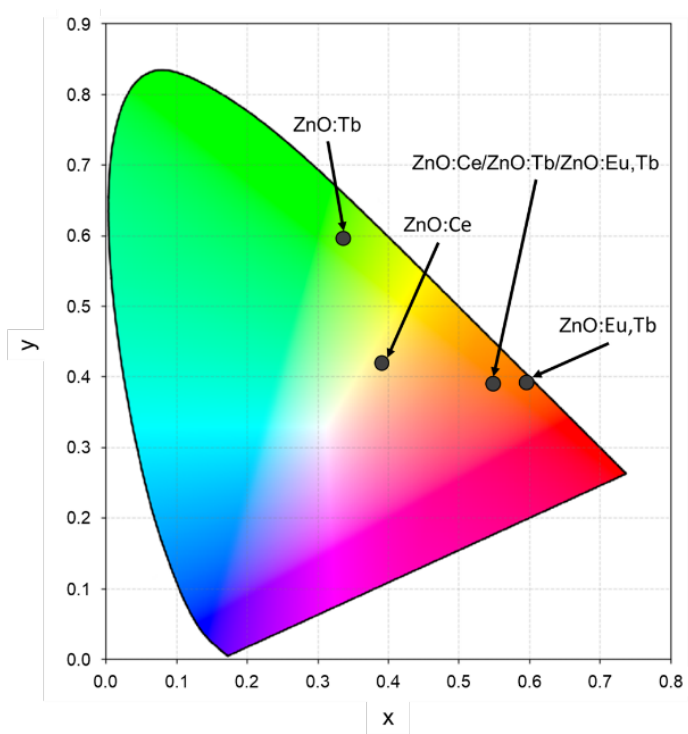

Figure 5. Location of the emission corresponding to each LED under study in the present work within the standard CIE 1931 chromatic diagram.

Indeed, from the chromatic diagram it becomes clear that blue emission from $\mathrm{Ce}$ ions has not yet been achieved or is highly quenched by the emission of $\mathrm{ZnO}$-defects. If blue emission from $\mathrm{Ce}^{3+}$ could be added to the system, the position of the multilayered sample would become even closer to the center. Therefore, aiming at obtaining an efficient RE-based RGB LED, it is necessary to improve the deposition of a light-emitting $\mathrm{Ce}^{3+}$-doped $\mathrm{ZnO}$-based layer whose emission can provide the blue component expected to be combined with the intense green $\left(\mathrm{Tb}^{3+}\right)$ and red $\left(\mathrm{Eu}^{3+}\right)$ hereby already demonstrated.

\section{Conclusion}

The devices hereby analyzed have proved the possibility of fabricating an RGB LED employing $\mathrm{ZnO}$ as host matrix for low concentration $\mathrm{RE}$ ions, using sputtering as the main deposition technique. For this purpose, three different $\mathrm{RE}$ elements have been explored, $\mathrm{Ce}, \mathrm{Tb}$ and $\mathrm{Eu}$, whose EL emission characteristics (determined by the electronic transitions when in a +3 valence state) respectively result in blue, green and red intense and narrow emissions.

Out of the three, $\mathrm{Tb}^{3+}$ and $\mathrm{Eu}^{3+}$ are optically active after the deposition and annealing processes underwent to achieve the device structure, and under the electrical excitation conditions employed for attaining EL devices emission; instead, Ce has only shown poor EL emission and only under high stress conditions. The analysis and characterization via TEM and EDX, as well as the electrical conduction mechanisms analysis, point towards the agglomeration of Ce ions in the layer to be larger than in the case of $\mathrm{Tb}$ and $\mathrm{Eu}$, affecting their local stoichiometry and therefore carrier transport and emission properties. Nonetheless, the results for $\mathrm{Ce}$ are still promising, and future test and characterization of the $\mathrm{ZnO}: \mathrm{Ce}$ films controlling Ceconcentration and distribution are expected to improve their EL efficiency. The stack fabrication of the three films into a single device has also been tested, enabling the emission of the different optically-active centers present within the whole structure. As intended, their combined emission exhibits contribution from the three RE dopants, resulting in a shift towards the central region of the chromatic diagram. 
Overall, the hereby presented results reinforce the suitability of $\mathrm{ZnO}$ as an adequate matrix to host optically-active RE ions with intense and narrow emission, useful for fabricating full-color light sources for general lighting and other optoelectronics applications.

\section{Acknowledgements}

This work was financially supported by the Spanish Ministry of Economy and Competitiveness (Project No. TEC2016-76849-C2-1-R). J.L.F. acknowledges the subprogram "Ayudas para la Formación de Profesorado Universitario" (No. FPU16/06257) from the Spanish Ministry of Education, Culture and Sports for economical support. J.L.-V. acknowledges financial support from a "Juan de la Cierva" postdoctoral fellowship (IJCI-2017-33451) from the Spanish Ministry of Economy, Industry and Competitiveness. X.P., C.L., and C.G. are grateful to C. Frilay for his expertise in the maintenance of the sputtering setup used for the growth of the $\mathrm{ZnO}$ films. They also thank F. Lemarié for the TEM sample preparation by FIB.

\section{References}

[1] Özgür Ü, Alivov Y I, Liu C, Teke A, Reshchikov M A, Doğan S, Avrutin V, Cho S J and Morko H 2005 A comprehensive review of $\mathrm{ZnO}$ materials and devices J. Appl. Phys. $981-103$

[2] Klingshirn C F 2007 ZnO: Material, physics and applications ChemPhysChem 8782 803

[3] Rogers D J, Teherani F H, Sandana V E and Razeghi M $2010 \mathrm{ZnO}$ thin films and nanostructures for emerging optoelectronic applications Optoelectronic Integrated Circuits XII vol 7605, ed L A Eldada and E-H Lee (SPIE) p 76050K

[4] Laube J, Nübling D, Beh H, Gutsch S, Hiller D and Zacharias M 2016 Resistivity of atomic layer deposition grown $\mathrm{ZnO}$ : The influence of deposition temperature and postannealing Thin Solid Films 603 377-81

[5] Chen X L, Wang F, Geng X H, Zhang D K, Wei C C, Zhang X D and Zhao Y 2012 Natively textured surface Al-doped ZnO-TCO layers with gradual oxygen growth for thin film solar cells via magnetron sputtering Appl. Surf. Sci. 258 4092-6

[6] Rahman F 2019 Zinc oxide light-emitting diodes: a review Opt. Eng. 581

[7] Prucnal S, Wu J, Berencén Y, Liedke M O, Wagner A, Liu F, Wang M, Rebohle L, Zhou S, Cai H and Skorupa W 2017 Engineering of optical and electrical properties of $\mathrm{ZnO}$ by non-equilibrium thermal processing: The role of zinc interstitials and zinc vacancies J. Appl. Phys. 122

[8] Janotti A and Van De Walle C G 2009 Fundamentals of zinc oxide as a semiconductor Reports Prog. Phys. 72126501

[9] Fan J C, Sreekanth K M, Xie Z, Chang S L and Rao K V 2013 p-Type ZnO materials: Theory, growth, properties and devices Prog. Mater. Sci. 58 874-985

[10] Zeng H, Duan G, Li Y, Yang S, Xu X and Cai W 2010 Blue Luminescence of ZnO Nanoparticles Based on Non-Equilibrium Processes: Defect Origins and Emission Controls Adv. Funct. Mater. 20 561-72

[11] Duan L, Wang P, Wei F, Zhang W, Yao R and Xia H 2014 Electroluminescence of ZnO nanorods embedded in a polymer film Solid State Commun. 200 14-6 
[12] Kenyon A J 2002 Recent developments in rare-earth doped materials for optoelectronics Prog. Quantum Electron. 26 225-84

[13] Khan L U and Khan Z U 2018 Rare earth luminescence: Electronic spectroscopy and applications Handbook of Materials Characterization (Cham: Springer International Publishing) pp 345-404

[14] Gaponenko N V., Sergeev O V., Borisenko V E, Pivin J C, Skeldon P, Thompson G E, Hamilton B, Misiewicz J, Bryja L, Kudrawiec R, Stupak A P and Stepanova E A 2001 Terbium photoluminescence in polysiloxane films Mater. Sci. Eng. B Solid-State Mater. Adv. Technol. 81 191-3

[15] Heikenfeld J, Garter M, Lee D S, Birkhahn R and Steckl A J 1999 Red light emission by photoluminescence and electroluminescence from Eu-doped GaN Appl. Phys. Lett. 75 1189-91

[16] Luo Q, Wang L S, Guo H Z, Lin K Q, Chen Y, Yue G H and Peng D L 2012 Blue luminescence from $\mathrm{Ce}$-doped $\mathrm{ZnO}$ thin films prepared by magnetron sputtering $\mathrm{Appl}$. Phys. A Mater. Sci. Process. 108 239-45

[17] Anand V, Sakthivelu A, Kumar K D A, Valanarasu S, Ganesh V, Shkir M, Kathalingam A and AlFaify S 2018 Novel rare earth Gd and Al co-doped ZnO thin films prepared by nebulizer spray method for optoelectronic applications Superlattices Microstruct. 123 $311-22$

[18] Komuro S, Katsumata T, Morikawa T, Zhao X, Isshiki H and Aoyagi Y $20001.54 \mathrm{Mm}$ Emission Dynamics of Erbium-Doped Zinc-Oxide Thin Films Appl. Phys. Lett. 76 3935-7

[19] Lluscà M, López-Vidrier J, Antony A, Hernández S, Garrido B and Bertomeu J 2014 Up-conversion effect of Er- and Yb-doped ZnO thin films Thin Solid Films 562 456-61

[20] Röder R, Geburt S, Zapf M, Franke D, Lorke M, Frauenheim T, da Rosa A L and Ronning C 2019 Transition Metal and Rare Earth Element Doped Zinc Oxide Nanowires for Optoelectronics Phys. Status Solidi Basic Res. 256 1-12

[21] Ishii M, Komuro S, Morikawa T and Aoyagi Y 2001 Local structure analysis of an optically active center in Er-doped ZnO thin film J. Appl. Phys. 89 3679-84

[22] Li J, Zalloum O H Y, Roschuk T, Heng C L, Wojcik J and Mascher P 2008 Light emission from rare-earth doped silicon nanostructures Adv. Opt. Technol. 2008

[23] Umamaheswari D, Jamalaiah B C, Sasikala T, Chengaiah T, Kim I G and Rama Moorthy L 2012 Photoluminescence and decay behavior of Tb 3+ ions in sodium fluoroborate glasses for display devices J. Lumin. 132 1166-70

[24] Sorokina I T 2007 Crystalline Mid-Infrared Lasers Solid-State Mid-Infrared Laser Sources vol 351 (Berlin, Heidelberg: Springer Berlin Heidelberg) pp 262-358

[25] Jovanović D J, Antić Ž, Krsmanović R M, Mitrić M, Crossed D Signorcrossed D Signević V, Bártová B and Dramićanin M D 2013 Annealing effects on the microstructure and photoluminescence of Eu 3+-doped GdVO4 powders Opt. Mater. (Amst). 35 1797-804

[26] Polman A 1997 Erbium implanted thin film photonic materials J. Appl. Phys. 82 1-39

[27] Takahei K, Taguchi A, Horikoshi Y and Nakata J 1994 Atomic configuration of the ErO luminescence center in Er-doped GaAs with oxygen codoping J. Appl. Phys. 76 43329

[28] Ford G M and Wessels B W 1996 1.54 ??M Electroluminescence From Erbium Doped 
Gallium Phosphide Diodes Mater. Res. Soc. Symp. - Proc. 422 345-50

[29] Jambois O, Berencen Y, Hijazi K, Wojdak M, Kenyon A J, Gourbilleau F, Rizk R and Garrido B 2009 Current transport and electroluminescence mechanisms in thin $\mathrm{SiO} 2$ films containing Si nanocluster-sensitized erbium ions J. Appl. Phys. 106063526

[30] Ramírez J M, Ferrarese Lupi F, Jambois O, Berencén Y, Navarro-Urrios D, Anopchenko A, Marconi A, Prtljaga N, Tengattini A, Pavesi L, Colonna J P, Fedeli J M and Garrido B 2012 Erbium emission in MOS light emitting devices: from energy transfer to direct impact excitation Nanotechnology 23125203

[31] He X, Liu X, Li R, Yang B, Yu K, Zeng M and Yu R 2016 Effects of local structure of Ce3+ ions on luminescent properties of Y3A15O12:Ce nanoparticles Sci. Rep. 6 1-11

[32] Mishra S, Kshatri D S, Khare A, Tiwari S and Dwivedi P K 2016 SrS:Ce3+ thin films for electroluminescence device applications deposited by electron-beam evaporation deposition method Mater. Lett. 183 191-6

[33] Luo L, Huang F Y, Guo G J, Tanner P A, Chen J, Tao Y T, Zhou J, Yuan L Y, Chen S Y, Chueh Y L, Fan H H, Li K F and Cheah K W 2012 Efficient doping and energy transfer from $\mathrm{ZnO}$ to $\mathrm{Eu} 3+$ ions in Eu 3+-doped $\mathrm{ZnO}$ nanocrystals J. Nanosci. Nanotechnol. 12 2417-23

[34] Davesnne C, Ziani A, Labbé C, Marie P, Frilay C and Portier X 2014 Energy transfer mechanism between terbium and europium ions in zinc oxide and zinc silicates thin films Thin Solid Films 553 33-7

[35] Blázquez O, López-Vidrier J, López-Conesa L, Busquets-Masó M, Estradé S, Peiró F, Hernández S and Garrido B 2016 Structural and optical properties of $\mathrm{Al}-\mathrm{Tb} / \mathrm{SiO} 2$ multilayers fabricated by electron beam evaporation J. Appl. Phys. 120135302

[36] Frieiro J L, Blázquez O, López-Vidrier J, López-Conesa L, Estradé S, Peiró F, Ibáñez J, Hernández S and Garrido B 2018 Green Electroluminescence of Al/Tb/Al/SiO 2 Devices Fabricated by Electron Beam Evaporation Phys. status solidi 2151700451

[37] Borkovska L V., Khomenkova L, Korsunska O, Kolomys O, Strelchuk V, Sabov T, Venger E, Kryshtab T, Melnichuk O, Melnichuk L, Guillaume C, Labbe C and Portier X 2019 Influence of annealing on luminescence and energy transfer in $\mathrm{ZnO}$ multilayer structure co-doped with Tb and Eu Thin Solid Films 692137634

[38] Petersen J, Brimont C, Gallart M, Schmerber G, Gilliot P, Ulhaq-Bouillet C, Rehspringer J L, Colis S, Becker C, Slaoui A and Dinia A 2010 Correlation of structural properties with energy transfer of Eu-doped $\mathrm{ZnO}$ thin films prepared by sol-gel process and magnetron reactive sputtering J. Appl. Phys. 107 1-7 